\title{
Planejamento de Caminho Seguro com Espuma Probabilística para um Robô Móvel
}

\author{
Luís B. P. Nascimento* Vitor G. Santos* Diego S. Pereira* \\ Daniel H. S. Fernandes* William C. Ribeiro* \\ Dennis Barrios-Aranibar ${ }^{* *}$ Raquel E. Patiño-Escarcina ** \\ Pablo J. Alsina* Joelson C. Rocha Júnior ${ }^{* * *}$ \\ * Departamento de Engenharia de Computação e Automação, \\ Universidade Federal do Rio Grande do Norte, RN, Brasil (e-mail: \\ \{lbruno,vitorgaboardi\}@ufrn.edu.br,pablo@dca.ufrn.br). \\ ** Departamento de Ingeniería Eléctrica y Electrónica, Universidad \\ Católica San Pablo, Peru (e-mail: dbarrios@ucsp.edu.pe) \\ *** Programa de Pós-Graduação em Engenharia Elétrica, Universidade \\ Federal do Espirito Santo, ES, Brasil
}

\begin{abstract}
This paper presents an application of the Probabilistic Foam Method in the path planning of a differential drive mobile robot. The major advantage of this method is the creation of safe path that is significantly far from the obstacles based in the radius of a structure called bubble. In this application, the new bubble is computed based in the information from workspace rather than the configuration space. The proposed methodology was tested in a virtual environment using a path tracking algorithm that computes the angular velocity that moves the robot to the desired position, which is the path created. Simulations shows that the system is able to plan free obstacle paths with a high degree of safety.

Resumo: Este artigo apresenta aplicação do método Espuma Probabilística no planejamento de caminho de um robô móvel com acionamento diferencial. Esse método tem como principal vantagem a criação de caminhos seguros considerando um certo grau de afastamento entre a região de obstáculos com base no raio de uma estrutura chamada bolha. Para essa aplicação, foi calculada uma nova bolha com informação do espaço de trabalho, não sendo necessário o cálculo explícito da região de C-obstáculos. A metodologia empregada foi testada por meio de simulações utilizando um controlador de caminho que calcula a velocidade angular que o robô deve ter para alcançar e seguir o caminho criado. Foi possível constatar que a estratégia empregada foi capaz de planejar caminhos livre de obstáculos e com um certo grau de segurança.
\end{abstract}

Keywords: Autonomous Robotics; Path Planning; Probabilistic Foam; Bubbles of Free Space; Differential Robot.

Palavras-chaves: Robótica Autônoma; Planejamento de Caminho; Espuma Probabilística; Bolhas do Espaço Livre; Robô Diferencial.

\section{INTRODUÇÃO}

O planejamento de caminho é um dos principais problemas da robótica autônoma em geral e possui um papel fundamental no projeto de robô móveis (Hassani et al., 2018). Técnicas de planejamento de caminho calculam uma sequência de configurações (poses e orientações) que o robô móvel deve assumir para atingir um determinado objetivo, isto é, sair de uma configuração inicial $q_{\text {inicial }}$, até uma configuração final $q_{\text {final }}$, sem colidir com possíveis obstáculos (Svestka and Overmars, 1998).

Dentre os planejadores encontrados na literatura, é possível destacar os métodos probabilísticos que se baseiam na captura de amostras aleatórias do espaço de configuração, sendo assim, capazes de construir uma represen-

\footnotetext{
* O presente trabalho foi realizado com apoio da Coordenação de Aperfeiçoamento de Pessoal de Nível Superior - Brasil (CAPES) Código de Financiamento 001.
}

tação aproximada da topologia do espaço livre, o que os tornam computacionalmente viáveis (Latombe, 1991; LaValle, 2006). Dentre os diversos métodos probabilísticos de planejamento, é possível citar o Mapa de Rotas Probabilístico (Probabilistic Roadmaps) (Kavraki et al., 1996) e a Árvore Aleatória de Exploração Rápida (RapidlyExploring Random Tree - RRT) (Lavalle, 1998; Lavalle et al., 2000) como os mais populares.

Um dos pontos importantes do planejamento é garantir que o robô seja capaz de realizar manobras de maneira segura. Alguns planejadores, ao calcular o caminho, eventualmente geram rotas perigosas para o robô móvel, como uma grande proximidade das paredes e obstáculos (Yu et al., 2011), podendo acarretar em colisões no mundo real. Para corrigir esse problema, algumas modificações nos métodos de planejamento ou de sensoriamento são realizadas (Lambert and Gruyer, 2003), visando aumentar a margem de segurança para que não haja eventuais colisões. 
A Espuma Probabilística (Probabilistic Foam Method PFM) é um método de planejamento que além de prover um caminho livre de obstáculos, garante uma região de segurança para manobrabilidade do robô. O planejador PFM visa representar de forma aproximada o espaço livre por meio de bolhas conectadas. As bolhas se propagam no espaço livre formando uma estrutura chamada espuma probabilística que representa uma cobertura aproximada do espaço livre, característica similar aos métodos de decomposição aproximada em células. A propagação da espuma ocorre de forma similar à propagação em frente de onda, com as bolhas formando uma árvore de busca, similar à árvore do método RRT. Em Nascimento et al. (2018) o PFM foi aplicado no planejamento dos movimentos de um sistema robótico que apresenta juntas rotacionais. Uma característica interessante da metodologia empregada para a modelagem da bolha foi que o cálculo explícito da região C-obstáculos não foi um requisito para o planejamento.

Neste artigo é proposto uma utilização do método Espuma Probabilística no planejamento de caminhos seguros para um robô móvel, sem a necessidade de calcular a região de obstáculos no espaço de configurações por meio da modelagem de uma nova bolha. Para testar a metodologia proposta, simulações foram realizadas aplicando o planejamento para um robô móvel com tração diferencial. Uma estratégia de controle foi utilizada para prover os movimentos ao robô.

As próximas seções estão organizadas como segue: $\mathrm{Na}$ seção 2 é apresentado o método de planejamento Espuma probabilística e suas principais características. Na seção seguinte é apresentada a metodologia para o cálculo da nova bolha para o PFM. Na seção 4, é descrita a modelagem do robô móvel no qual foi aplicado o planejamento. Na seção 5 é apresentada a estratégia de controle utilizada. Na seção 6 são apresentados os resultados e finalmente, na seção 7, são apresentadas as conclusões.

\section{O MÉTODO DA ESPUMA PROBABILÍSTICA}

Introduzido por Silveira and Alsina (2016), a Espuma Probabilística (Probabilistic Foam Method - PFM) é um método de planejamento de caminho capaz de garantir uma região de segurança para manobrabilidade por meio de uma região no espaço livre $C_{f}$ chamada bolha. Uma bolha $b$ de raio $r$, centrada na configuração $q_{c} \in C_{f}$ é uma $n$ - bola definida como:

$$
b=b\left(q_{c}, r\right)=\left\{q / d\left(q, q_{c}\right)<r\right\}
$$

sendo $d\left(q, q_{c}\right)$ a métrica utilizada no espaço de configurações $C$ e $r$ é a distância mínima entre $q_{c}$ e a região de obstáculos $C_{o}$ no espaço de configurações, ou seja:

$$
r=\min _{q^{\prime} \in C_{o}} d\left(q^{\prime}, q_{c}\right)
$$

Dessa forma, uma bolha centrada em $q_{c}$ pode expandir-se de seu centro até o obstáculo mais próximo.

\subsection{A propagação da espuma}

A espuma probabilística $E$ é uma coleção de bolhas sobrepostas que se propagam por $C_{f}$ por meio da expansão de bolhas filhas centradas na superfície de outras bolhas (bolhas mães). Considerando que a superfície de uma bolha é uma $(n-1)$-esfera, o número $N$ máximo de bolhas filhas que podem ser geradas por uma bolha mãe é:

$$
N=K\left(\left\lfloor\frac{r}{r_{\min }}\right\rfloor\right)^{n-1}
$$

onde $r_{\text {min }}$ é uma constante que indica o menor raio aceitável para uma bolha e $r$ é o raio da bolha mãe. A constante $K$ define o número máximo de bolhas filhas que uma bolha com raio $r_{\min }$ pode gerar.

O processo de propagação da espuma inicia-se a partir de uma bolha inicial $b_{\text {inicial }}$ expandida sobre a configuração inicial $q_{\text {inicial }}$ onde em sua superfície são geradas as bolhas filhas (Figura 1a). Uma bolha filha só é admissível se respeitar o critério de raio mínimo, $r \geqslant r_{\text {min }}$, e se ela não estiver centrada numa região coberta por outra bolha na espuma (Figura 1b). Todas as bolhas válidas são organizadas numa fila por ordem de expansão, sendo que, nessa mesma ordem, a nova bolha mãe é escolhida (Figura 1c). Esse processo de propagação ocorre até que uma nova bolha $b_{\text {final }}$ englobe a configuração final $q_{\text {final, }}$ e assim o método finaliza (Figura 1d).

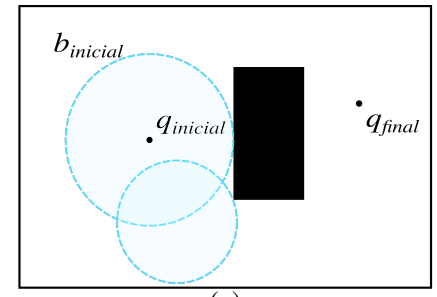

(a)

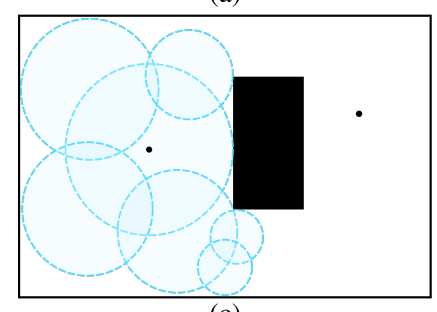

(c)

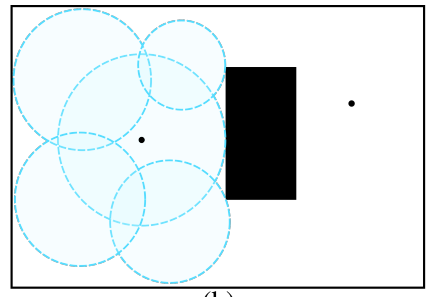

(b)

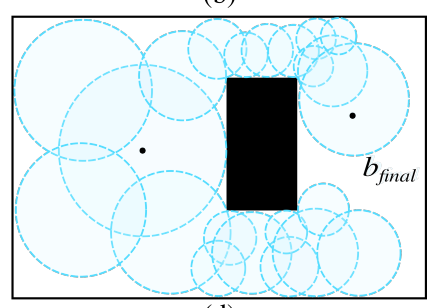

(d)
Figura 1. Propagação da espuma probabilística.

Com a espuma formada, é possível realizar uma busca entre as bolhas partindo da bolha final e seguindo a relação de parentesco até a configuração inicial, formando uma estrutura chamada Rosário. A partir do Rosário é possível extrair um caminho ligando o centro das bolhas, como ilustra a Figura 2.

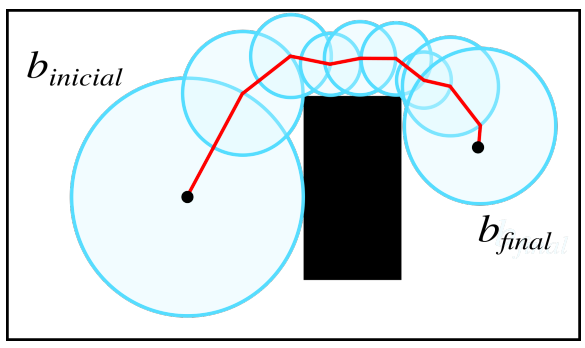

Figura 2. Modelo da bolha do espaço livre. 
O rosário é uma importante estrutura pois garante uma restrição de segurança para o caminho: Se o caminho atravessa todos os portais (região localizada dentro da região de interseção entre duas bolhas), é garantido que caminho está numa região do espaço livre.

Um pseudocódigo do método PFM é apresentado em Algoritmo 1.

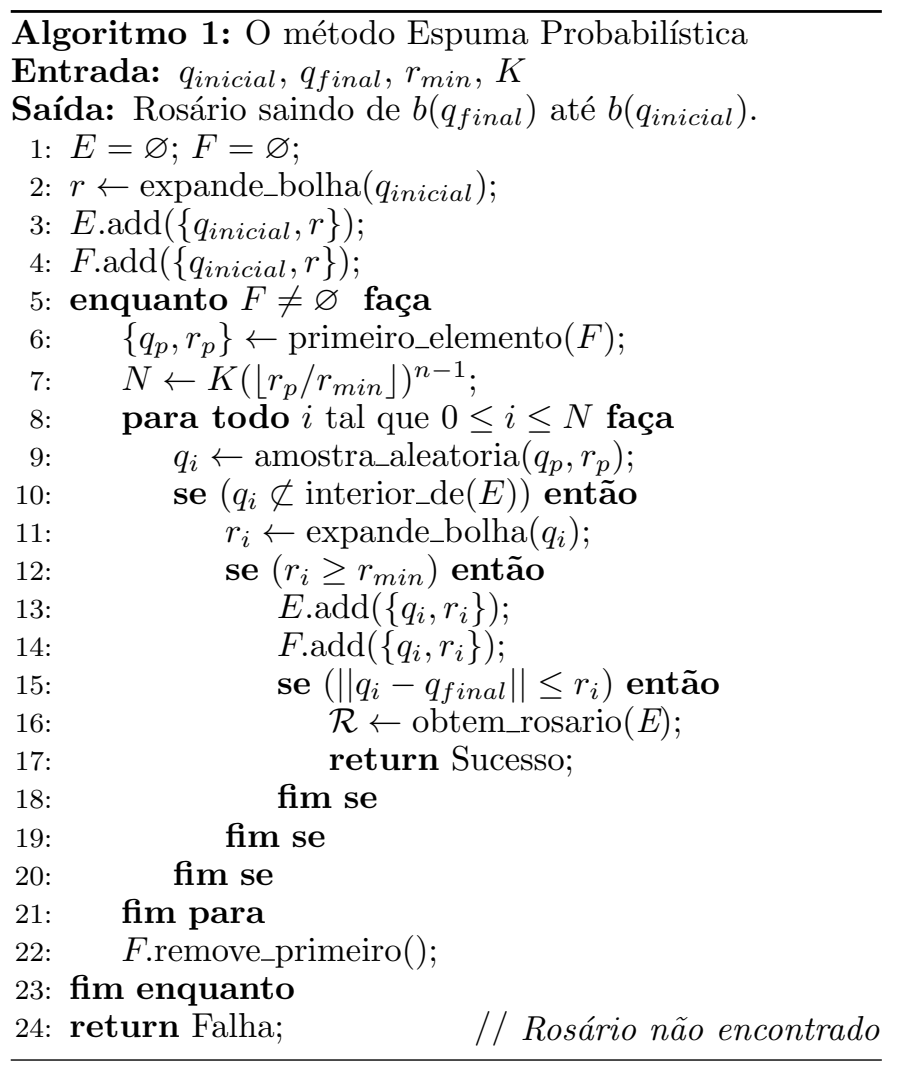

\section{MODELAGEM DA NOVA BOLHA PARA UM ROBO MÓVEL}

Como mostrado na seção anterior, a bolha calculada pelo PFM necessita do cálculo explícito da região de obstáculos no espaço de configurações $\mathcal{C}_{o}$. Dessa maneira, uma nova abordagem para o cálculo da bolha é apresentada, inspirada no conceito de bolhas do espaço livre. Esse conceito foi introduzido por Quinlan (1995).

A bolha do espaço livre é definida como uma região volumétrica em $\mathcal{C}$, calculada por meio da distância entre o robô e os obstáculos no espaço de trabalho $\mathcal{W}_{o}$. Considere que um robô centrado na configuração $q$ é deslocado para uma configuração $p$ qualquer. Se a distância desse deslocamento é menor que a distância entre o robô e a região $\mathcal{W}_{o}$, não há colisão.

Para garantir que não haja colisão para um robô com tração diferencial, considera-se que um cilindro de raio $r_{b}$ cobre todo o robô móvel. Assim, a nova bolha $B(q)$ é calculada com base na distância $d_{t}$ entre esse cilindro e a região $\mathcal{W}_{o}$, ou seja:

$$
B(q)=\left\{p:\|q-p\| \leqslant d_{t}\right\}
$$

A Figura 3 ilustra a expansão da nova bolha $B(q)$ para um robô móvel centrado na configuração $q$.

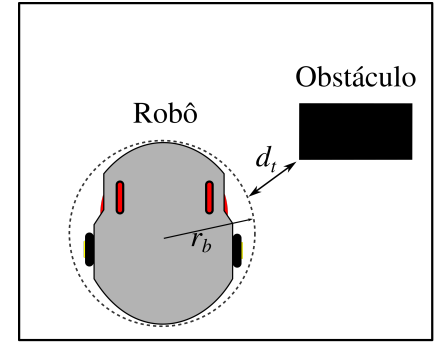

(a)

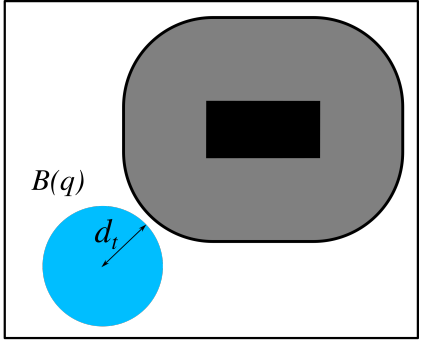

(b)
Figura 3. Modelo da bolha $B(q)$ para um robô móvel centrado na configuração $q$.

A Figura 3a apresenta um robô no espaço de trabalho e a Figura $3 \mathrm{~b}$ apresenta a bolha $B$ gerada para esse robô. A partir dessa nova bolha, o PFM fica habilitado a planejar caminhos para um robô móvel sem a necessidade do cálculo explícito da região de obstáculos.

\section{CONTROLADOR DE CAMINHO}

Com o objetivo de fazer com que o robô móvel com acionamento diferencial siga o caminho gerado pelo método da Espuma Probabilística, foi necessário realizar a implementação de um controlador de caminho. Nesse trabalho, optou-se pela escolha de um algoritmo denominado Pure Persuit (Coulter, 1992), visto que o mesmo possui uma simples implementação e atende aos requisitos de desempenho desse trabalho.

A lei de controle desse algoritmo se baseia, inicialmente, em inserir um arco que conecte a posição atual do robô a um ponto no caminho de referência que esteja a uma distância $L$, chamada de distância lookahead (Paden et al., 2016). A Figura 4 ilustra a geometria dessa configuração, onde o arco de raio $R$ está representado em vermelho, a distância $L$ em azul e o caminho de referência em verde.

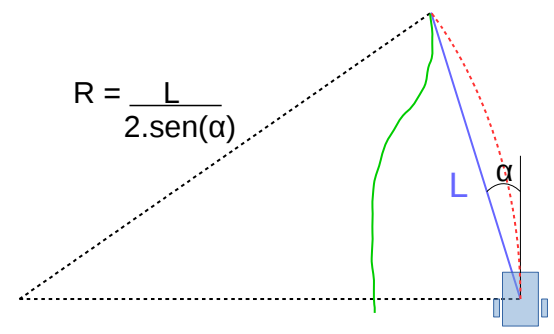

Figura 4. Geometria do algoritmo do Pure Persuit

Considere que o robô possua as coordenadas $\left(x_{r}, y_{r}, \theta\right)$, onde $\theta$ representa o ângulo de orientação do robô em relação ao referencial fixo no espaço de trabalho e as coordenadas $\left(x_{r e f}, y_{r e f}\right)$ tal que $\left\|\left(x_{r e f}, y_{r e f}\right)-\left(x_{r}, y_{r}\right)\right\|=$ $L$. Dessa forma, o cálculo de $\alpha$, mostrado na Figura 4, é dado por:

$$
\alpha=\arctan \left(\frac{y_{r e f}-y_{r}}{x_{r e f}-x_{r}}\right)-\theta
$$

Assim, a curvatura do arco $(\kappa)$ é dada por: 


$$
\kappa=\frac{1}{R}=\frac{2 \cdot \operatorname{sen}(\alpha)}{L}
$$

E, para uma velocidade linear constante $(v)$, tem-se:

$$
\omega=\frac{2 \cdot v \cdot \operatorname{sen}(\alpha)}{L}
$$

no qual $\omega$ representa a velocidade angular necessária para que o robô saia de sua posição atual e vá para a posição no caminho de referência que está a uma distância $L$. Essa trajetória é feita através do arco mostrado na Figura 4.

É interessante analisar como o valor de $L$, que é um parâmetro definido pelo programador, modifica a forma como o caminho será percorrido. Um alto valor faz com que o robô tenda a convergir ao caminho de forma gradual e suave, ignorando pontos do caminho que estão próximos e "olhando" para pontos mais distantes. Já com um baixo valor, o robô se move rapidamente em direção ao caminho, porém, podendo resultar em algumas oscilações em torno do mesmo. A Figura 5 ilustra essas duas configurações.

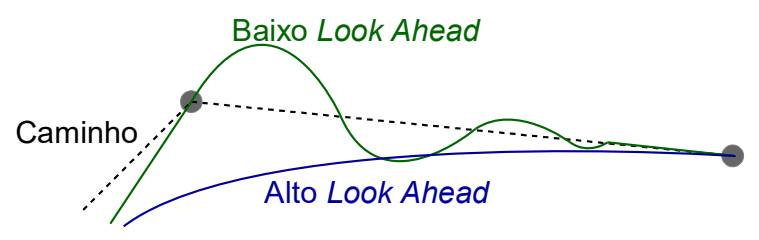

Figura 5. Configuração do sistema para dois valores da distância lookahead.

Como a proposta do controlador desenvolvido nesse projeto foi fazer com que o robô siga estritamente o caminho para evitar colisões com obstáculos, utilizou-se um baixo valor da distância lookahead.

\section{MODELAGEM CINEMÁTICA DE UM ROBÔ COM ACIONAMENTO DIFERENCIAL}

Na seção anterior, definiu-se a velocidade angular do robô móvel para alcançar o caminho gerado pelo método da Espuma Probabilística. Entretanto, precisamos utilizar essa informação para obter as velocidades angulares da roda direita $\omega_{D}$ e da roda esquerda $\omega_{E}$, visto que essas são as variáveis necessárias para acionar o robô móvel. Dessa forma, realiza-se a modelagem cinemática de um robô móvel, onde obtém-se uma equação que relaciona a estrutura física do robô com os parâmetros de velocidade.

É interessante ressaltar que o modelo cinemático não considera as forças dinâmicas que atuam sobre o robô. Nesse sentido, as estratégias de controle são baseadas apenas em modelos cinemáticos, isto é, a parte mecânica do sistema robótico responde de forma instantânea ao sinal de controle gerado pelo controlador (Vieira, 2006).

A Figura 6 ilustra o robô móvel com acionamento diferencial modelado nesse trabalho. Destaca-se que o mesmo possui duas rodas traseiras fixas, coaxiais, independentes e tracionadas. Os parâmetros utilizados para esquematizar o robô são apresentados a seguir:

- $a$ : comprimento do eixo;

- $r_{D}$ : raio da roda direita;

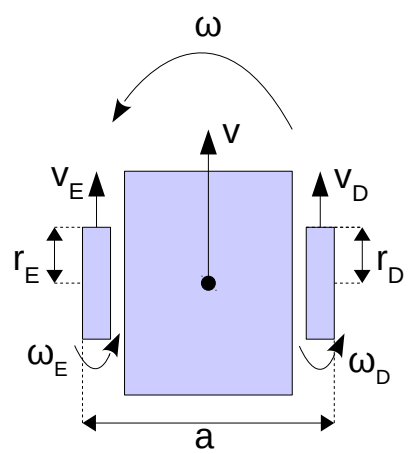

Figura 6. Robô Móvel com Acionamento Diferencial.

- $r_{E}$ : raio da roda esquerda;

- $\omega$ : velocidade angular do robô;

- $\omega_{D}$ : velocidade angular da roda direita;

- $\omega_{E}$ : velocidade angular da roda esquerda;

- $v$ : velocidade linear do robô;

- $v_{D}$ : velocidade linear da roda direita;

- $v_{E}$ : velocidade linear da roda esquerda;

A Figura 7 mostra a representação do raio de giro $r_{G}$ do robô móvel considerando movimentos infinitesimais.

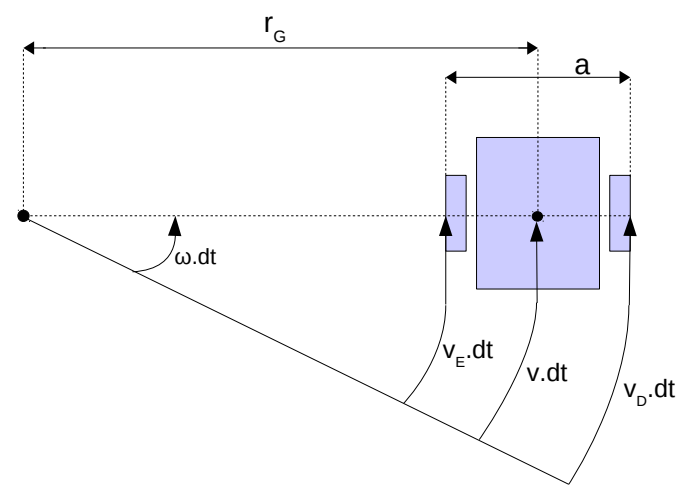

Figura 7. Representação do raio de giro do robô.

Assim, as velocidades angulares das rodas do robô são dadas por:

$$
\begin{gathered}
v_{D} \cdot d t=\omega(r+a / 2) \cdot d t \\
v_{E} \cdot d t=\omega(r-a / 2) \cdot d t \\
v_{D}+v_{E}=\omega_{D} \cdot r_{D}+\omega_{E} \cdot r_{E}=2 \cdot \omega \cdot r=2 . v \\
v_{D}-v_{E}=\omega_{D} \cdot r_{D}-\omega_{E} \cdot r_{E}=\omega \cdot b
\end{gathered}
$$

Isolando $v$ na Equação 10 e $\omega$ na Equação 11 em função de $\omega_{D}$ e $\omega_{E}$ e expressando a relação dessas variáveis em uma matriz, tem-se:

$$
\left[\begin{array}{c}
v \\
w
\end{array}\right]=\left[\begin{array}{cc}
\left(r_{D} / 2\right) & \left(r_{E} / 2\right) \\
\left(r_{D} / a\right) & -\left(r_{E} / a\right)
\end{array}\right] \cdot\left[\begin{array}{c}
w_{D} \\
w_{E}
\end{array}\right]
$$

Como os parâmetros a serem controlados são $w_{D}$ e $w_{E}$, podemos rearranjar a Equação 12, obtendo: 


$$
\left[\begin{array}{l}
w_{D} \\
w_{E}
\end{array}\right]=\left[\begin{array}{cc}
\left(1 / r_{D}\right) & \left(a / 2 . r_{D}\right) \\
\left(1 / r_{E}\right) & -\left(a / 2 . r_{E}\right)
\end{array}\right] \cdot\left[\begin{array}{c}
v \\
w
\end{array}\right]
$$

Assim, por meio da velocidade linear $v$ e da velocidade angular $\omega$, que foram obtidos no controlador de caminho, é possível determinar as velocidades angulares de cada roda de forma que o robô siga o caminho livre de obstáculos obtido pelo do método da Espuma Probabilística.

\section{RESULTADOS}

A fim de demonstrar a metodologia apresentada, modelamos um ambiente para a realização da simulação por meio do software Virtual Robot Experimentation Platform (Rohmer et al., 2013) ou simplesmente V-REP, como mostra a Figura 8. Além disso, utilizamos um robô de tração diferencial para seguir o caminho planejado pelo método Espuma Probabilística.

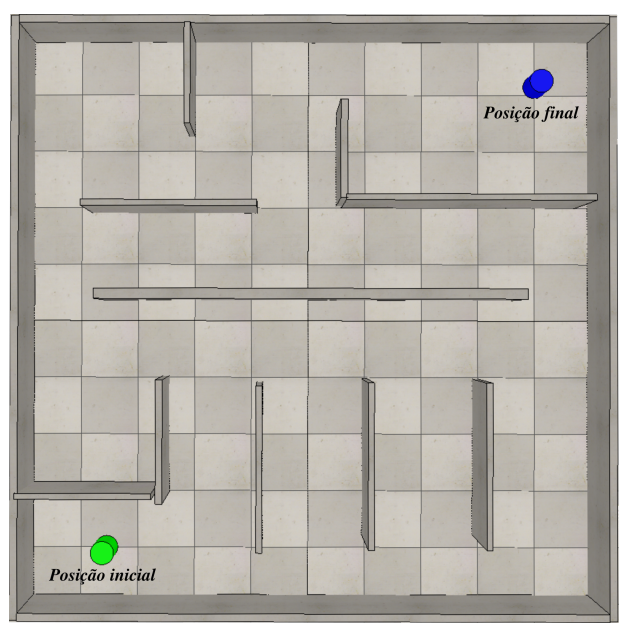

Figura 8. Ambiente criado no V-REP para a simulação.

Os parâmetros do PFM foram definidos como $r_{\min }=0.01$ e $K=3$ para a simulação. Esses parâmetros foram definidos de maneira empírica por meio de uma série de execuções, de forma que o método sempre encontrasse um caminho. A espuma, o rosário e o caminho resultantes do planejamento para o mapa apresentado, são mostrados nas Figuras 9 e 10 .

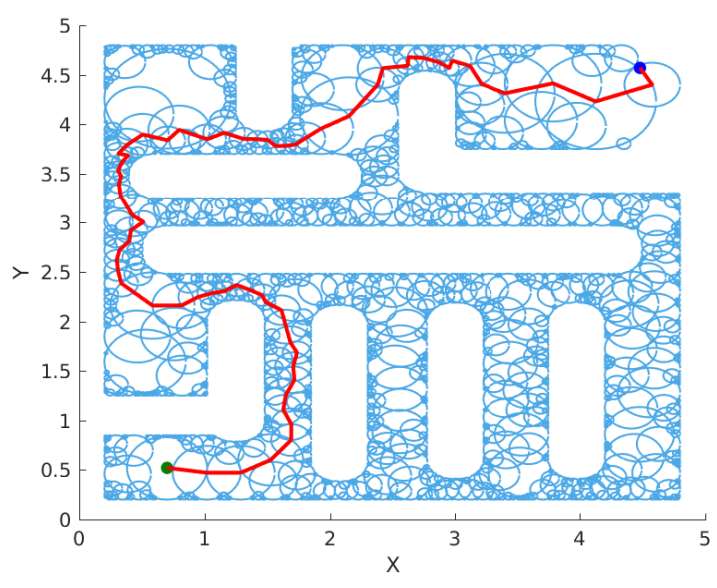

Figura 9. Espuma probabilística gerada.

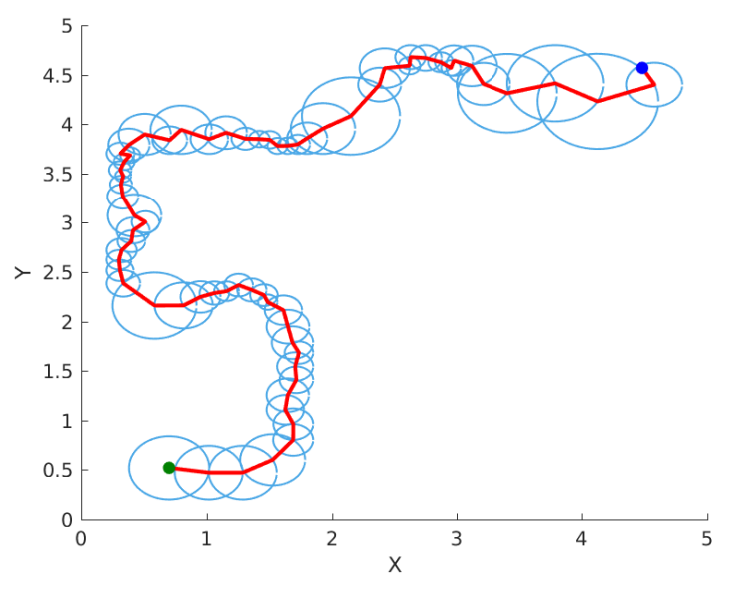

Figura 10. Rosário extraído e caminho planejado.

É possível perceber que a espuma probabilística se propagou pelo espaço livre, contornando a região de obstáculos, sem que $\mathcal{C}_{o}$ tenha sido calculado de maneira explícita anteriormente. A cobertura aproximada realizada pela espuma viabiliza encontrar caminhos entre qualquer ponto coberto pelas bolhas no mapa até a configuração inicial. Se for considerado executar o PFM partindo da configuração final, será possível extrair caminhos partindo de qualquer ponto coberto até a configuração final.

A trajetória realizada pelo robô é mostrada na Figura 11. A linha vermelha representa o caminho obtido pelo planejamento com a Espuma Probabilística a ser seguido pelo robô e a linha azul apresenta a trajetória percorrida. O controlador Pure Persuit aplicado permitiu a execução de uma trajetória próxima do caminho gerado, utilizando um valor da distância lookahead $L=0,2$ metros.

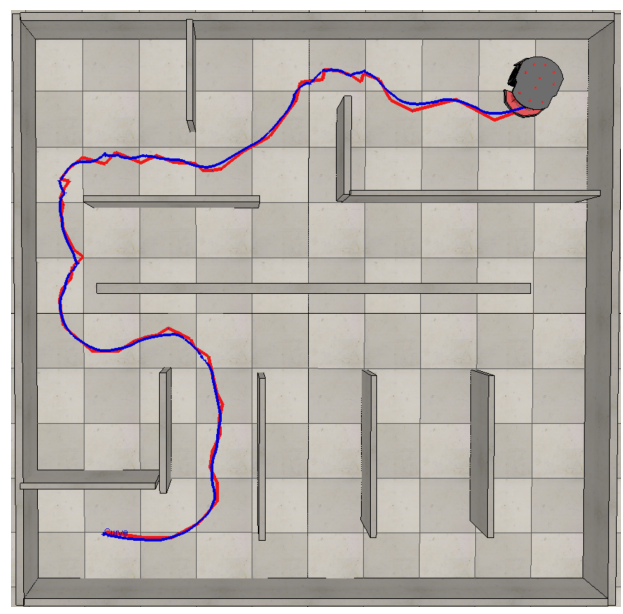

Figura 11. Simulação com a trajetória do robô.

Pode ser observado que o caminho obtido no planejamento apresenta algumas curvas bruscas que não respeitam as restrições não-holonômicas do robô. Entretanto, o controlador de caminho utilizado com os parâmetros descritos possibilitou uma melhor adaptação ao caminho percorrido.

A Figura 12 apresenta a trajetória realizada pelo robô durante a simulação. Observa-se que essa trajetória está inserida dentro da região das bolhas do rosário. Isso permitiu que todas as configurações assumidas pelo robô fossem 


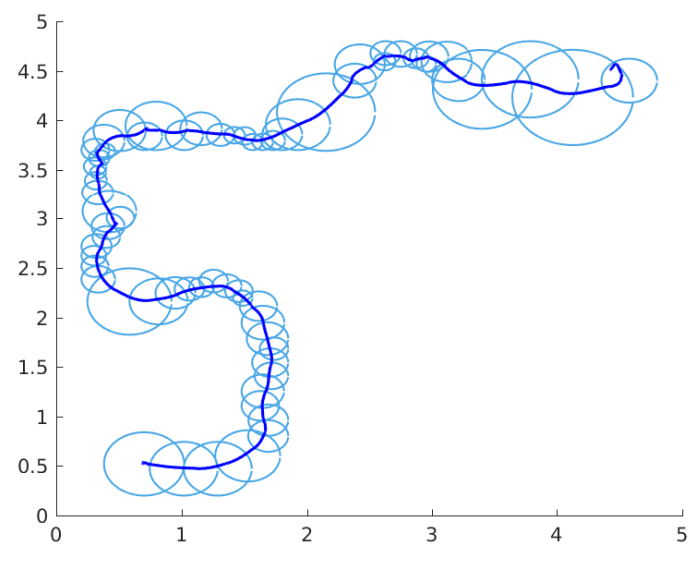

Figura 12. Rosário extraído e caminho percorrido.

seguras, ou seja, livres de colisão. Além disso, é possível perceber a existência de uma margem de manobrabilidade, enfatizando a viabilidade do método para o cenário proposto.

\section{CONCLUSÕES}

Esse trabalho apresentou a aplicação do método de planejamento de caminho Espuma Probabilística para um robô móvel, onde uma nova bolha foi calculada com base nas informações de distância no espaço de trabalho. Tal característica é uma vantagem em relação a abordagem original do PFM, a qual necessita da realização do cálculo explícito da região de obstáculos no espaço de configurações.

A abordagem utilizada no cálculo da bolha foi interessante pois permitiu que robôs rotacionais fossem modelados como robôs circulares, onde a orientação é desconsiderada, o que facilitou o processo de planejamento.

A utilização do método de controle Pure Persuit permitiu que o robô de tração diferencial pudesse seguir o caminho planejado. Além disso, com a escolha adequada de um parâmetro exclusivo desse controlador, a distância lookahead, uma melhor adequação da trajetória ao rosário foi possibilitada, garantindo mais segurança.

Finalmente, perspectivas futuras dessa pesquisa envolvem a geração de caminhos mais suaves e adequados ao robô, assim como a realização de experimentos em ambientes reais com o objetivo de validar a metodologia apresentada.

\section{REFERÊNCIAS}

Coulter, R.C. (1992). Implementation of the pure pursuit path tracking algorithm. Technical report, CarnegieMellon UNIV Pittsburgh PA Robotics INST.

Hassani, I., Maalej, I., and Rekik, C. (2018). Robot path planning with avoiding obstacles in known environment using free segments and turning points algorithm. Mathematical Problems in Engineering, 2018.

Kavraki, L.E., Svestka, P., Latombe, J.., and Overmars, M.H. (1996). Probabilistic roadmaps for path planning in high-dimensional configuration spaces. IEEE Transactions on Robotics and Automation, 12(4), 566-580. doi:10.1109/70.508439.
Lambert, A. and Gruyer, D. (2003). Safe path planning in an uncertain-configuration space. In 2003 IEEE International Conference on Robotics and Automation (Cat. No. 03CH37422), volume 3, 4185-4190. IEEE.

Latombe, J.C. (1991). Robot Motion Planning. Springer US, Boston, MA. doi:10.1007/978-1-4615-4022-9.

Lavalle, S.M. (1998). Rapidly-exploring random trees: A new tool for path planning.

Lavalle, S.M., Kuffner, J.J., and Jr. (2000). RapidlyExploring Random Trees: Progress and Prospects. In Algorithmic and Computational Robotics: New Directions, 293-308.

LaValle, S.M. (2006). Planning Algorithms. Cambridge University Press, Cambridge ; New York. OCLC: ocm65301992.

Nascimento, L.B.P., Pereira, D.S., Sanca, A.S., Eugenio, K.J.S., Fernandes, D.H.S., Alsina, P.J., Araujo, M.V., and Silva, M.R. (2018). Safe path planning based on probabilistic foam for a lower limb active orthosis to overcoming an obstacle. In 2018 Latin American Robotic Symposium, 2018 Brazilian Symposium on Robotics (SBR) and 2018 Workshop on Robotics in Education (WRE), 413-419. doi:10.1109/LARS/SBR/WRE.2018. 00080 .

Paden, B., Čáp, M., Yong, S.Z., Yershov, D., and Frazzoli, E. (2016). A survey of motion planning and control techniques for self-driving urban vehicles. IEEE Transactions on intelligent vehicles, 1(1), 33-55.

Quinlan, S. (1995). Real-Time Modification of CollisionFree Paths. Doctoral dissertation, Stanford University Stanford, CA.

Rohmer, E., Singh, S.P., and Freese, M. (2013). V-rep: A versatile and scalable robot simulation framework. In 2013 IEEE/RSJ International Conference on Intelligent Robots and Systems, 1321-1326. IEEE.

Silveira, Y.S. and Alsina, P.J. (2016). A New Robot Path Planning Method Based on Probabilistic Foam. In 2016 XIII Latin American Robotics Symposium and IV Brazilian Robotics Symposium (LARS/SBR), 217-222. doi:10.1109/LARS-SBR.2016.43.

Svestka, P. and Overmars, M.H. (1998). Probabilistic Path Planning. In M. Thoma (ed.), Robot Motion Planning and Control, volume 229 of Lecture Notes in Control and Information Sciences, 255-304. Springer Berlin Heidelberg, Berlin, Heidelberg. doi:10.1007/ BFb0036069.

Vieira, F.C. (2006). Controle dinâmico de robôs móveis com acionamento diferencial. Master's thesis, Universidade Federal do Rio Grande do Norte.

Yu, C.J., Chen, Y.H., and Wong, C.C. (2011). Path planning method design for mobile robots. In SICE Annual Conference 2011, 1681-1686. IEEE. 